\title{
Characterization of the polarization properties of biological tissues with fiber-based Mueller-matrix optical coherence tomography
}

Shuliang Jiao, Tseng-Ming Hsieh, Jun Ai, Milos Todorovic, George Stoica, et al.

Shuliang Jiao, Tseng-Ming Hsieh, Jun Ai, Milos Todorovic, George Stoica, Lihong V. Wang, "Characterization of the polarization properties of biological tissues with fiber-based Mueller-matrix optical coherence tomography," Proc. SPIE 5319, Laser Interaction with Tissue and Cells XV, (1 July 2004); doi: $10.1117 / 12.531498$

SPIE. Event: Biomedical Optics 2004, 2004, San Jose, CA, United States 


\title{
Characterization of the polarization properties of biological tissues with fiber-based Mueller-matrix optical coherence tomography
}

\author{
Shuliang Jiao ${ }^{\mathrm{a}}$, Tseng-Ming Hsieh ${ }^{\mathrm{a}}$, Jun $\mathrm{Ai}^{\mathrm{a}}$, Milos Todorovic ${ }^{\mathrm{a}}$ \\ George Stoica ${ }^{\mathrm{b}}$, and Lihong V. Wang ${ }^{\mathrm{a}}$ \\ ${ }^{a}$ Optical Imaging Laboratory, Department of Biomedical Engineering, Texas A\&M University, 3120 \\ TAMU, College Station, TX 77843-3120 \\ ${ }^{\mathrm{b}}$ Department of Pathobiology, Texas A\&M University, College Station, TX 77843-5547
}

\begin{abstract}
A fast scanning fiber-based system of Mueller-matrix optical coherence tomography was built to characterize the polarization properties of biological tissues with high spatial resolution. A polarization modulator with its fast-axis oriented at $45^{\circ}$ in the source arm of the Michelson interferometer, driven by a sinusoidal wave, was used to continuously modulate the incident polarization states of both the sample and the reference arms. Two detection channels were used to detect the horizontal and vertical polarization components of the interference signals, which were used to calculate the roundtrip Jones matrix of the sample. The roundtrip polarization parameters of the sample were calculated from the measured Jones matrix. The system was successfully tested for both standard optical polarization elements and various types of biological samples.
\end{abstract}

Keywords: optical coherence tomography, Mueller-matrix, Jones Matrix polarization

\section{INTRODUCTION}

Polarization-sensitive optical coherence tomography (PS-OCT) ${ }^{1}$ can provide polarization-based contrast in addition to the amplitude-based contrast provided by conventional OCT. In polarimetry, the polarization properties of a nondepolarizing sample can be completely characterized by either a Jones matrix or a Mueller matrix. Upon measuring the Jones or Mueller matrix, various polarization parameters can be calculated. Mueller-matrix $\mathrm{OCT}^{2,3}$ is a branch of PSOCT that can provide complete characterization of the polarization properties of biological tissues with high spatial resolution by measuring the depth-resolved Jones or Mueller matrix. In previous versions of multi-channel Muellermatrix OCT, two light sources with orthogonal polarization states and two detection channels were used to acquire the Jones matrix of a sample with a single measurement. In this paper, we report on a new Mueller-matrix OCT system that uses a single light source and a polarization modulator, which continuously modulates the incident polarization states for both the sample and the reference arms. The system is capable of acquiring the Jones matrix of a sample in vivo with a single measurement.

\section{EXPERIMENTAL SETUP}

A schematic of the experimental system is shown in figure 1. A superluminescent diode (SLD, center wavelength: 1.3 um, $3 \mathrm{~dB}$ bandwidth: $70 \mathrm{~nm}$, output power: $10 \mathrm{~mW}$ ) is used as the low coherence light source. The source light is vertically polarized by a linear polarizer. A polarization modulator driven by a sinusoidal wave is used to continuously modulate the source polarization state. The fast axis of the polarization modulator is oriented at $45^{\circ}$. To satisfy the requirement for calculating the Jones matrix ${ }^{2,3}$ and to preserve the spatial resolution of OCT, the modulation must be fast enough to ensure that several cycles of polarization states are applied to each pixel of the OCT image. The reference arm and the sample arm are composed of single mode optical fibers. A linear polarizer (LP) oriented at $-45^{\circ}$ is placed in the reference arm to set the reference polarization state. A grating-based rapid scanning optical delay line (RSOD) is used for the depth scan. The combined sample and reference light is split into horizontal and vertical polarization components by a polarizing beam splitter (PBS). The two orthogonal polarization components of the interference signal are detected by two photodiodes, PDH and PDV, respectively. The detected interference signals are high-pass filtered by an analog 
filter (Standford SR 650), digitized by a 12-bit data acquisition board (NI DAQ, PCI-MIO-16E), and processed by a computer. The system is capable of acquiring a full Jones matrix image of $2 \mathrm{~mm} \times 2 \mathrm{~mm}$ with a frame rate as high as $2 \mathrm{fps}$.

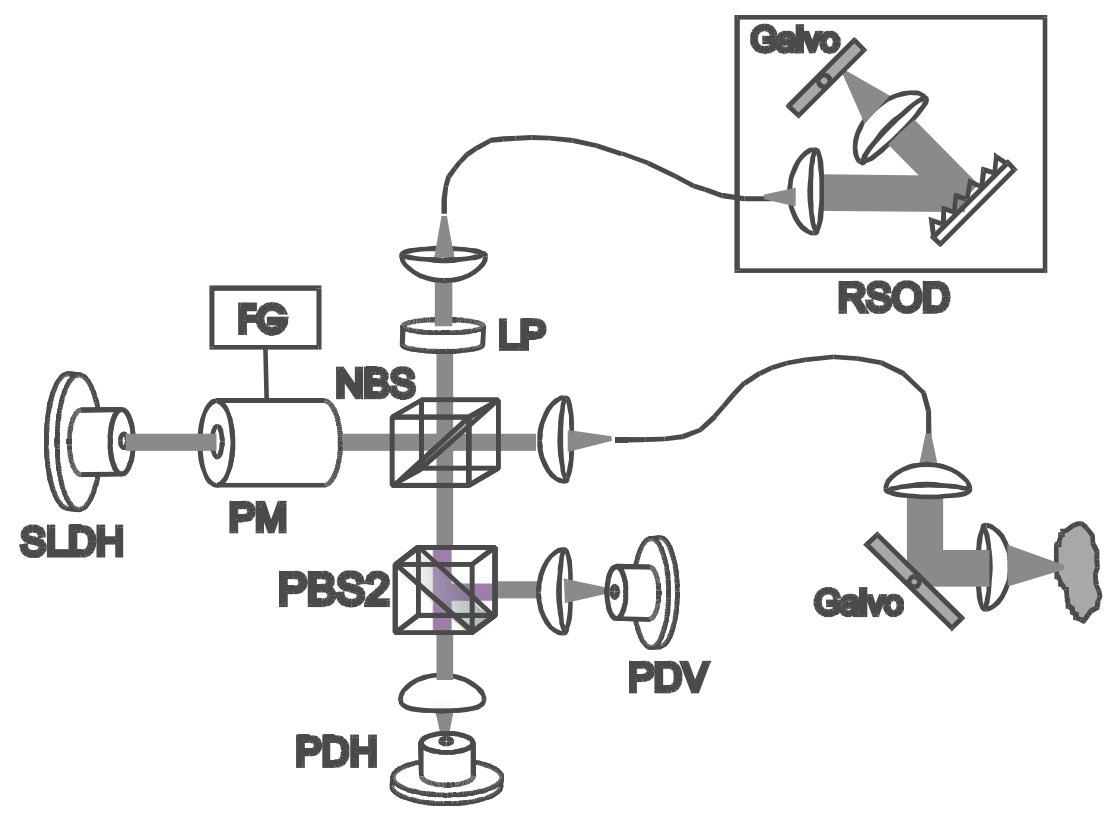

Figure 1. Schematic of the experimental system. SLDH: SLD, horizontally polarized; PM: polarization modulator; FG: function generator; NBS: non-polarizing beam splitter; PBS2: polarizing beam splitter; SMF: single-mode optical fiber; PDH and PDV: photodiodes $\mathrm{H}$ and $\mathrm{V}$ polarization. RSOD: rapid scanning optical delay line; LP: $45^{\circ}$ linear polarizer.

\section{ALGORITHM FOR JONES MATRIX CALCULATION}

The Jones matrix of the polarization modulator $\mathbf{J}_{\mathrm{m}}$ can be expressed as

$$
\mathbf{J}_{m}(\varphi, \pi / 4)=\left[\begin{array}{cc}
\cos \varphi / 2 & i \sin \varphi / 2 \\
i \sin \varphi / 2 & \cos \varphi / 2
\end{array}\right]
$$

where $\varphi$ is the phase retardation of the polarization modulator. The incident Jones vector $E_{i}$ for both the sample and the reference arms can be expressed as

$$
\mathbf{E}_{i}=\left[\begin{array}{c}
i \sin (\varphi / 2) \\
\cos (\varphi / 2)
\end{array}\right]
$$

The output Jones vector $E_{o}$ of the sample arm can be expressed as

$$
\mathbf{E}_{o}=\mathbf{J}_{T} \mathbf{E}_{i},
$$

where $\mathbf{J}_{T}$ is the round-trip Jones matrix of the sample arm.

The output Jones vector of the reference arm can be expressed as

$$
\mathbf{E}_{r e f}=\left[\begin{array}{c}
E_{r h} \\
-E_{r v}
\end{array}\right] \exp (-i \varphi / 2),
$$

where $E_{r h}$ and $E_{r v}$ have the same phase $\varphi_{r}$ and are functions of the round-trip Jones matrix of the single mode optical fiber in the reference arm. 
The detected signals in the horizontal and vertical channels can be expressed as

$$
\begin{aligned}
& I_{x}=I_{0 x}+\tilde{I}_{x} \\
& I_{y}=I_{0 y}+\tilde{I}_{y}
\end{aligned}
$$

where $\tilde{I}_{x}$ and $\tilde{I}_{y}$ are the interference signals in the horizontal and vertical channels; and $I_{0 x}$ and $I_{0 y}$ are the noninterference signals. When the polarization modulator is driven by a sinusoidal wave:

$$
\varphi=2.405 \sin \omega_{m} t
$$

where $\omega_{m}$ is the angular frequency of the driving wave, we can derive

$$
\begin{aligned}
\tilde{I}_{x} & =\left|E_{r h}\right| \cdot|J(1,2)-J(1,1)| \cos \left(\bar{k} z+\varphi_{r}-\varphi_{x 1}\right) \\
& +\left|E_{r h}\right| \cdot|J(1,1)+J(1,2)| \cos \left(\bar{k} z+\varphi_{r}-\varphi_{x 2}\right) \sum 2 J_{2 l}(2.405) \cos \left(2 l \omega_{m} t\right) \\
& +\left|E_{r h}\right| \cdot|J(1,1)+J(1,2)| \sin \left(\bar{k} z+\varphi_{r}-\varphi_{x 2}\right) \sum 2 J_{2 l+1}(2.405) \sin \left[(2 l+1) \omega_{m} t\right], \\
\tilde{I}_{y} & =\left|E_{r v}\right| \cdot|J(2,2)-J(1,2)| \cos \left(\bar{k} z+\varphi_{r}+\pi-\varphi_{y 1}\right) \\
& +\left|E_{r v}\right| \cdot|J(1,2)+J(2,2)| \cos \left(\bar{k} z+\varphi_{r}+\pi-\varphi_{y 2}\right) \sum 2 J_{2 l}(2.405) \cos \left(2 l \omega_{m} t\right) \\
& +\left|E_{r v}\right| \cdot|J(1,2)+J(2,2)| \sin \left(\bar{k} z+\varphi_{r}+\pi-\varphi_{y 2}\right) \sum 2 J_{2 l+1}(2.405) \sin \left[(2 l+1) \omega_{m} t\right]
\end{aligned} .
$$

where $\bar{k}$ is the center wave number of the light source in the vacuum; $\mathrm{z}$ the path length difference between the reference and sample arms; $J(1,1), J(1,2), J(2,1)$, and $J(2,2)$ are the elements of $\mathbf{J}_{T} ; \varphi_{x 1}, \varphi_{x 2}, \varphi_{y 1}$ and $\varphi_{y 2}$ are the phases of $J(1,2)-J(1,1), J(1,1)+J(1,2), J(2,2)-J(1,2)$ and $J(1,2)+J(2,2)$, respectively; $J_{2 l+1}$ and $J_{2 l}$ the Bessel functions of the first kind of the orders of $2 l+1$ and $2 l$.

The elements of $\mathbf{J}_{T}$ can be calculated from two frequency components:

$$
\begin{aligned}
J(1,1) & =\left[-\tilde{I}_{x}^{*}(\bar{k} z)+\tilde{I}_{x}\left(\omega_{m} t-\bar{k} z\right) / J_{1}(2.405)\right] /\left|E_{r h}\right|, \\
J(1,2) & =\left[\tilde{I}_{x}^{*}(\bar{k} z)+\tilde{I}_{x}\left(\omega_{m} t-\bar{k} z\right) / J_{1}(2.405)\right] /\left|E_{r h}\right|, \text { or } \\
J(1,2) & =\left[\tilde{I}_{y}^{*}(\bar{k} z)-\tilde{I}_{y}\left(\omega_{m} t-\bar{k} z\right) / J_{1}(2.405)\right] /\left|E_{r v}\right|, \\
J(2,2) & =\left[-\tilde{I}_{y}^{*}(\bar{k} z)-\tilde{I}_{y}\left(\omega_{m} t-\bar{k} z\right) / J_{1}(2.405)\right] /\left|E_{r v}\right| .
\end{aligned}
$$

As a result, the Jones matrix of the sample arm can be calculated from two measured frequency terms in the horizontal and vertical detection channels. One term has a frequency that is determined by the depth scan (the carrier frequency), and the other term has the beat frequency between the modulation frequency of the polarization modulator and the carrier frequency. Other frequency terms can also be used in the calculation of $\mathbf{J}_{T}$.

A single-mode optical fiber alters the polarization state of the guided light due to its inherent birefringence. Furthermore, the birefringence varies with the bending and twisting of the fiber, which can result in dynamic distortions in the polarization images. Since the sample arm is composed of the sampling fiber and the sample, a dynamic calibration technique is required to eliminate the polarization distortions in $\mathbf{J}_{T}$ that are introduced by the sampling fiber. The measured roundtrip Jones matrix $\mathbf{J}_{T}$ can be expressed as

$$
\mathbf{J}_{T}=\mathbf{J}_{f 1}^{T} \mathbf{J}_{s 2} \mathbf{J}_{f 1} .
$$

where $\mathbf{J}_{f 1}$ and $\mathbf{J}_{s 2}$ are the one-way and round-trip Jones matrices of the sampling fiber and the sample, respectively. To eliminate $\mathbf{J}_{f 1}$ from $\mathbf{J}_{T}, \mathbf{J}_{f 1}$ needs to be calculated from the round-trip Jones matrix of the sampling fiber $\mathbf{J}_{f 2}$, 
which is measured from the reflecting surface of the sample. We have proved that $\mathbf{J}_{f 1}$ can be calculated from $\mathbf{J}_{f 2}$ with a free parameter. ${ }^{4}$ We denote $\mathbf{J}_{f l 1}$ as the calculated $\mathbf{J}_{f 1}$ from $\mathbf{J}_{f 2}$. The calibration process can be expressed as

$$
\mathbf{J}_{s c 2}=\mathbf{J}_{f l 1}^{-1} \mathbf{J}_{T} \mathbf{J}_{f l 1}^{-1}
$$

where $\mathbf{J}_{s c 2}$ is the calibrated result for $\mathbf{J}_{s 2}$. The round-trip Jones matrix of the sample can the be expressed as ${ }^{4}$

$$
\begin{aligned}
& \mathbf{J}_{s 2}=R(\gamma) \mathbf{J}_{s c 2} R(-\gamma) \\
& R(\gamma)=\left[\begin{array}{cc}
\cos \gamma & \sin \gamma \\
-\sin \gamma & \cos \gamma
\end{array}\right] .
\end{aligned}
$$

As a result, the calibration is equivalent to rotating the sample along the axis of the incident light by an angle $\gamma$. This rotation does not change the amplitudes of either the birefringence or the diattenuation. The orientation of the birefringence can be calculated with an offset $\gamma, \theta_{s 2}=\theta_{s c 2}-\gamma$, where $\theta_{s c 2}$ is the orientation of the fast eigenvector of $\mathbf{J}_{s c 2}$. The offset $\gamma$ depends on the parameters of the sampling fiber only. This offset is a constant in a frame of image as long as the parameters of the sampling fiber are kept constant during the image acquisition of each frame, which is the case when the fast lateral scanning of OCT does not move the sampling fiber. Therefore, a relative distribution of the orientation of the birefringence can be retrieved. If the parameters of the sampling fiber are varied among the A scans, which is true when the lateral scanning in OCT does move the sampling fiber, $\gamma$ will differ among the A lines. In this case, if the orientation of the birefringence of the surface layer is constant, or known a priori, or if a known thin retarder is attached to the sample as the first layer, $\gamma$ can be eliminated. In either case, the round-trip retardation of the sample,

$\varphi_{s 2}$ can be calculated exactly by ${ }^{5}$

$$
\varphi_{s 2}=2 \cos ^{-1}\left\{\frac{1}{2} \frac{\left|\operatorname{tr} \mathbf{J}_{s 2}+\operatorname{det} \mathbf{J}_{s 2} /\right| \operatorname{det} \mathbf{J}_{s 2}\left|\operatorname{tr} \mathbf{J}_{s 2}^{*}\right|}{\left[\operatorname{tr}\left(\mathbf{J}_{s 2}^{*} \mathbf{J}_{s 2}\right)+2\left|\operatorname{det} \mathbf{J}_{s 2}\right|\right]^{1 / 2}}\right\},
$$

\section{EXPERIMENTAL RESULTS}

We tested the OCT system by imaging different types of biological samples in vivo. Fig. 2 shows the intensity image and the calculated retardation image of a piece of porcine tendon, respectively. The measured Jones matrix at the sample surface is used to eliminate the polarization effect of the sampling fiber. The sample beam was transversely scanned with a step size of $10 \mu \mathrm{m}$. The digitized interference signals were first band-pass filtered and then Hilbert transformed by the software to extract the analytical signals of each polarization component. Two-dimensional (2D) images were formed from the A-scans and then median filtered. Clear band structures can be seen in the retardation image. There is no such band structure present in the intensity image, which is the image of the $M_{00}$ element of the corresponding Mueller matrix. The band structure distributes quite uniformly in the measured region; therefore, the birefringence is also uniform in the measured area.
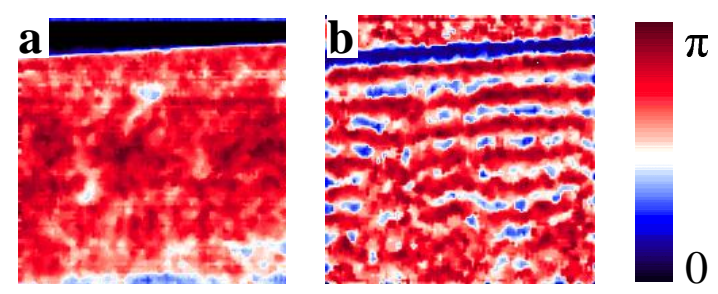

Fig. 2. The measured intensity image (a) and retardation image (b) for a piece of porcine tendon. The intensity image is on a logarithmic scale while the retardation image is on a linear scale. The color bar is for the retardation image. Image height: $1 \mathrm{~mm}$. 
Figure 3 shows the intensity and retardation images of the skin of a rat tail measured in vivo. After the rat was anesthetized (ketamine $60 \mathrm{mg} / \mathrm{kg}$, IM) and the hair of the tail was removed with hair remover lotion, the tail was scrubbed with glycerin. The intensity image and the retardation image reveal different information about the sample. The retardation image reveals the birefringence characteristics of the sample. In the retardation image, the dermal structures of the rat skin can be seen clearly.
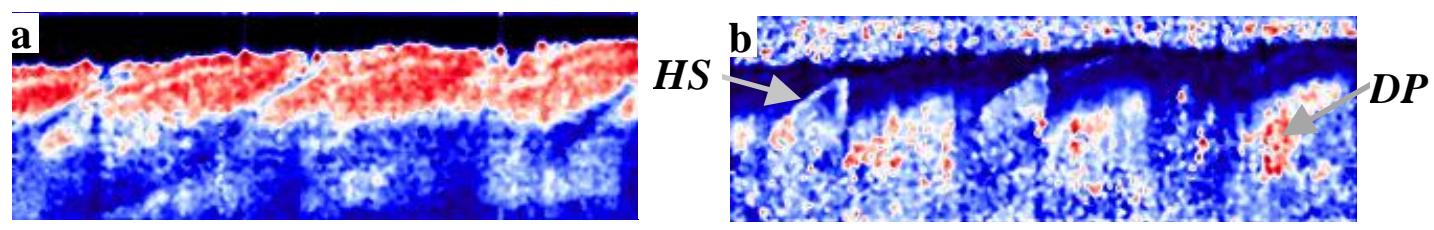

Fig. 3. The intensity image (a) and retardation image (b) for rat tail skin measured in vivo. The intensity image is on a logarithmic scale while the retardation image is on a linear scale. The color bar for the retardation image is the same as in Fig. 2. Image height: 1mm. HS: hair shaft; DP: dermal papilla.

In conclusion, we developed a novel fiber-based Mueller-matrix optical coherence tomography that enables the acquisition of a round-trip Jones matrix of biological samples using a single broadband source and a single measurement. A polarization modulator is used to continuously modulate the polarization state of the source. The Jones matrix of the sample arm can be calculated from two frequency terms in the two detection channels. By measuring the round trip Jones matrices of the sampling fiber from the reflecting surface of the sample, the fiber-induced polarization distortion can be eliminated and the Jones matrix of the sample can be extracted. The system was successfully applied to imaging biological samples.

\section{ACKNOWLEDGEMENT}

This project was sponsored in part by National Institutes of Health grants R21 EB00319-02 and R01 EB000712, by National Science Foundation grant BES-9734491, and by Texas Higher Education Coordinating Board grant 0005120063-2001. L. V. Wang's email address is LWang@tamu.edu.

\section{REFERENCES}

1. J. F. de Boer, T. E. Milner, M. J. C. van Gemert, and J. S. Nelson, "Two dimensional birefringence imaging in biological tissue by polarization-sensitive optical coherence tomography," Optics Letters, 22, 934-936 (1997).

2. S. Jiao and L. V. Wang, "Two-dimensional depth-resolved Mueller matrix of biological tissue measured with double-beam polarization-sensitive optical coherence tomography", Optics Letters 27(2), 101-103(2002).

3. S. Jiao and L. V. Wang, "Jones-matrix Imaging of biological tissues with quadraple-channel optical coherence tomography", J. of Biomedical Optics 7(3), 350-358 (2002).

4. S. Jiao, W. Yu, G. Stoica, and L. V. Wang, " Fiber-based Mueller optical coherence tomography", Optics Letters, 28(14), 12061208 (2003).

5. S. Y. Lu and R. A. Chipman, “Homogenous and inhomogenous Jones matrix”, J. Opt. Soc. Am. A 11, 766-772 (1987). 